\title{
PROPOSTA DE SISTEMATIZAÇÃO DAASSISTÊNCIA DE ENFERMAGEM PELAS TAXONOMIAS NANDA/NIC/NOC PARA O DIAGNOSTICO DE CONHECIMENTO DEFICIENTE
}

\author{
Juliana Cristina Pereira ${ }^{1}$, Rosamary Aparecida Garcia Stuchi ${ }^{2}$, Cristina Arreguy-Sena ${ }^{3}$
}

\begin{abstract}
RESUMO: Este trabalho configura-se como uma pesquisa clínica intervencionista que objetiva identificar diagnósticos de enfermagem emergentes, segundo taxonomia da North American Nursing Diagnosis Association; propor e implementar intervenções de enfermagem pertinentes ao diagnóstico “conhecimento deficiente” segundo taxonomia Nursing Intervention Classification e avaliar os resultados das intervenções implementadas a partir da taxonomia da Nursing Outcome Classification entre pessoas internadas na clínica médica de um hospital do interior de Minas Gerais. Foram identificados 84 diagnósticos nucleares no pré teste, sendo 25\% deles referentes ao “conhecimento deficiente”. Utilizouse folheto explicativo como recurso didático para implementar as intervenções e avaliaram-se as intervenções no pósteste, utilizando os indicadores propostos na taxonomia da Nursing Outcome Classification para o diagnóstico abordado. O teste de Wilcoxon evidenciou a significação das intervenções para aumentar o conhecimento dos participantes.

PALAVRAS-CHAVE: Enfermagem; Classificação; Diagnóstico de enfermagem; Cuidados de enfermagem.
\end{abstract}

\section{NURSING ASSISTANCE SYSTEMATIZATION PROPOSAL BY THE NANDA/NIC/NOC TAXONOMIES FOR THE DIAGNOSIS OF DEFFICIENT KNOWLEDGE}

\begin{abstract}
This work characterizes itself as an interventionist clinical research which aims at identifying emerging nursing diagnosis, following the taxonomy described in the North American Nursing Diagnosis Association (NANDA); proposing and implementing nursing interventions related to the diagnosis "Defficient knowledge" following the taxonomy described in the Nursing Intervention Classification (NIC) and evaluating the implemented intervention results by using the Nursing Outcome Classification (NOC) taxonomy among clinical patients in a hospital in the countryside of Minas Gerais. Eighty four nuclear diagnosis were identified in the pre-testing phase, $25 \%$ of which were related to "defficient knowledge". An explanatory pamphlet was used as a pedagogical resource to implement the interventions, and these were evaluated in the post-testing phase, by using the indicators proposed by the NOC taxonomy for the subject approached. Wilcoxon's Test brought evidence of the interventions' importance to increase participants' knowledge.
\end{abstract}

KEYWORDS: Nursing; Classification; Nursing diagnosis; Nursing care.

\section{PROPUESTA DE SISTEMATIZACIÓN DE LAASISTENCIA DE ENFERMERÍA POR LAS TAXONOMIAS NANDA/NIC/NOC PARA ELDIAGNÓSTICO DE CONOCIMIENTO DEFICIENTE}

RESUMEN: Este trabajo se configura como una investigación clínica intervencionista que objetiva identificar diagnósticos de enfermería emergentes, de acuerdo con la taxonomía de la North American Nursing Diagnosis Association; proponer e implementar intervenciones de enfermería referentes al diagnóstico “Conocimiento deficiente” de acuerdo con la taxonomía Nursing Intervention Classification y evaluar los resultados de las intervenciones aplicadas de la taxonomía de la Nursing Outcome Classification entre personas hospitalizadas en la clínica médica de un hospital del interior de Minas Gerais. Fueran identificados 84 diagnósticos nucleares en la pre-prueba, siendo 25\% de ellos referentes "conocimiento deficiente”. Se usó folleto explicativo como recurso didáctico para aplicar las intervenciones y fueron evaluadas las intervenciones en la pos-prueba, usando los indicadores propuestos en la taxonomía de la NOC para el diagnóstico abordado. La prueba de Wilcoxon mostró el significado de las intervenciones para aumentar el conocimiento de los participantes.

PALABRAS CLAVE: Enfermería; Clasificación; Diagnóstico de enfermería; Atención de enfermería.

${ }^{1}$ Enfermeira. Graduada pela Universidade Federal dos Vales do Jequetinhonha e Mucuri-UFVJM.

${ }^{2}$ Enfermeira. Doutora. Docente do Departamento de Enfermagem da UFVJM.

${ }^{3}$ Enfermeira. Doutora. Docente da Faculdade de Enfermagem da Universidade Federal de Juiz de Fora-UFJF.

Autor correspondente:

Rosamary Aparecida Garcia Stuchi

Universidade Federal dos Vales do Jequetinhonha e Mucuri

Rua José Rosa de Matos 162 - 39100-000 - Diamantina-MG, Brasil Recebido: 04/06/09

E-mail: meirestuchi@uol.com.br

Aprovado: 04/01/10

Cogitare Enferm 2010 Jan/Mar; 15(1):74-81 


\section{INTRODUÇÃO}

A Sistematização da Assistência de Enfermagem (SAE), regulada pela Lei do Exercício Profissional no 7489 de 25 de junho de 1986, é atividade privativa do enfermeiro. Visa assistir o ser humano por meio de ações específicas para promoção, prevenção, recuperação e reabilitação da saúde do indivíduo, da família e da comunidade ${ }^{(1)}$. Essa atividade compreende um conjunto de ações que, sistematizadas e interrelacionadas, possibilitam aplicar o método científico na prática assistencial, ao criar ligações entre o problema de saúde, a intervenção do enfermeiro e os resultados da assistência de enfermagem oferecida aos usuários ${ }^{(2-5)}$.

A SAE, que pode ser chamada de "processo de enfermagem", é composta pelas seguintes fases: coleta de dados; definição do problema; definição das intervenções; e avaliação dos resultados de enfermagem. Cabe destacar a variedade de terminologias usadas para expressar uma ou várias das etapas do processo de sistematização, a saber: 1) histórico, anamnese, ectoscopia, entrevista e/ou exame físico; 2) diagnóstico de enfermagem ou problema de enfermagem identificado a partir da etapa anterior, que aborda o julgamento clínico da resposta de um indivíduo, uma família ou uma comunidade diante de situações de doença, promoção da saúde ou do bemestar que estão em curso (reais) ou que podem se manifestar (potenciais) ${ }^{(4)}$; 3) metas terapêuticas e explicitação do nível de seu alcance em termos de tempo e de abrangência; 4) prescrições, aprazamento da prescrição, implantação da ação terapêutica, conduta terapêutica ou intervenções - fase de operacionalização da conduta terapêutica pertinente a uma abordagem individualizada do problema; 5) avaliação, evolução ou assessment - aferição da eficácia e eficiência das intervenções terapêuticas selecionadas para uma situação em foco ${ }^{(1-5)}$.

A estruturação do processo de enfermagem deve ser ancorada num referencial teórico da própria categoria $^{(2-3,5)}$. Horta ${ }^{(6)}$ descreveu sua fundamentação teórica embasada nas Necessidades Humanas Básicas de Maslow (ou teoria sobre a motivação humana hierarquizada) em cinco níveis de necessidades: 1) fisiológicas; 2) de segurança; 3) sociais; 4) de autoestima; 5) de autorrealização e, segundo a divisão proposta por João Mohana, necessidades de nível psicobiológico, psicossocial e psicoespiritual ${ }^{(4-6)}$.

Considerando que a responsabilidade do cuidar exige que as decisões sobre as intervenções propostas sejam fundamentadas na avaliação do estado de saúde do indivíduo, a sistematização é, em qualquer circunstância, primordial às suas ações, uma vez que se trata de um método eficiente de organização do pensamento para a tomada de decisões e consequente possibilidade de solução dos problemas detectados ${ }^{(2-3,7-8)}$.

A utilização de taxonomias para definir o problema de enfermagem tal como sugeridas pela North American Nursing Diagnosis Association (NANDA) $)^{(9)}$, intervenções de Enfermagem, pela Intervention Classification (NIC) ${ }^{(10)}$ e os resultados de enfermagem, pela Nursing Intervention Classification (NOC) ${ }^{(11)}$, podem exprimir a variedade de intervenções compatíveis com a prática de enfermagem internacional, e nortear a avaliação das intervenções implementadas. Elas se constituem em estratégias capazes de padronizar a linguagem, ou seja, um acordo sobre regras para utilização de determinados termos ${ }^{(2-3 ; 9-11)}$ dos componentes do processo de Enfermagem ${ }^{(2-3)}$. São também capazes de favorecer sistemas computadorizados de informação em enfermagem, bem como formar um conjunto uniformizado de dados, permitindo permuta internacional, bem como qualificar cuidados e avaliar a eficiência da Enfermagem.

Os diagnósticos de enfermagem são focos clínicos da profissão e a atividade diagnóstica aproxima profissionais e usuários, possibilitando-lhes conhecer melhor as respostas físicas e emocionais apresentadas durante o desenvolvimento do processo de trabalho do Enfermeiro.

A NANDA é exemplo de uma taxonomia que desenvolveu terminologias para descrever importantes julgamentos que os enfermeiros fazem quando provêem cuidados para indivíduos, famílias, grupos e comunidades. Tais julgamentos - ou diagnósticos são a base para a seleção de resultados e intervenções de enfermagem ${ }^{(9)}$.

Optamos por abordar o diagnóstico "conhecimento deficiente”, uma vez que ao retratar situações nas quais há "ausência ou deficiência de informação cognitiva relacionada a um tópico específico", é possível identificar comportamentos que dificultam a adesão ou o seguimento das recomendações terapêuticas, seja por falta de capacidade de recordar ou de interesse pelo conteúdo divulgado, seja por limitação no acesso às informações ou por interpretação errônea de informação ou, ainda, por limitação cognitiva, adquirindo significado 
específico dentro do processo de cuidar $^{(8: 171)}$. A identificação do referido diagnóstico, o acompanhamento da intervenção terapêutica selecionada para tratá-lo e a avaliação de seu impacto sobre o processo terapêutico constituem fatores relevantes e de impacto sobre o processo de cuidar em enfermagem.

Diante do exposto, este estudo se propõe a: identificar, segundo taxonomia NANDA, diagnósticos de enfermagem; propor e implementar, segundo taxonomia NIC, intervenções de enfermagem pertinentes ao diagnóstico "conhecimento deficiente"; e avaliar, a partir da taxonomia da NOC, entre pessoas internadas na clínica médica de um hospital do interior de Minas Gerais, o resultado das intervenções implementadas.

\section{MATERIAL E MÉTODOS}

Pesquisa clínica intervencionista ${ }^{(12)}$, realizada na unidade de clínica médica de um hospital de uma cidade do interior de Minas Gerais. Os dados foram coletados em outubro de 2008, sendo o processo de coleta antecedido por aprovação em Comitê de Ética em Pesquisa da Universidade Federal dos Vales do Jequitinhonha e Mucuri, sob o registro no 045/08. A amostra não probabilística por intencionalidade ${ }^{(12: 47)}$ foi composta por 25 pessoas que estavam internadas em uma enfermaria da clínica médica, escolhidas sob os seguintes critérios de inclusão: pessoas maiores que 18 anos, e que concordaram em participar mediante a assinatura do Termo de Consentimento Livre e Esclarecido, respeitados os preceitos da Resolução 196/ 96 sobre pesquisa com seres humanos.

O instrumento de coleta de dados foi estruturado conforme a proposta de Horta ${ }^{(5-6)}$, contendo os dados de entrevista e o exame físico. Usou-se a linha de raciocínio de Risner ${ }^{(13-14)}$ para que fossem identificados os diagnósticos nucleares dentro do sistema de problemas emergentes e, posteriormente, registrados na taxonomia da NANDA ${ }^{(9)}$.

Tendo como referência o componente dos fatores relacionados na taxonomia da NANDA, foram selecionadas as intervenções na taxonomia NIC para o diagnóstico "conhecimento deficiente" e, com base nas evidências clínicas (sinais e sintomas), ou seja, no componente das características definidoras da taxonomia da NANDA, foram identificados os critérios para avaliar os resultados segundo a taxonomia da NOC ${ }^{(2 ; 10-11)}$. Operacionalmente, a investigação foi estruturada em duas etapas: 1) coleta de dados para levantamento dos diagnósticos de enfermagem e aplicação das intervenções de enfermagem, segundo taxonomia da NIC; 2) avaliação dos resultados de enfermagem, segundo taxonomia da NOC.

Na primeira etapa (fase piloto), foi apresentado um roteiro preliminar para abordar o "conhecimento deficiente". O fato de ter sido construído predominantemente na modalidade descritiva, fez com que a rotina fosse modificado em sua formatação. Em sua versão final, o material de apoio didático (folheto explicativo com ilustrações coloridas), formatado com códigos ilustrativos contendo mensagem simples e de fácil entendimento, favoreceu e subsidiou as explanações e as orientações correspondentes ao conteúdo das intervenções de enfermagem realizadas. O núcleo conceitual das abordagens foi estruturado em referencial científico a respeito da temática. $\mathrm{O}$ instrumento de coleta de dados foi antecedido, em sua versão final, para validação da forma e do conteúdo, por cinco enfermeiras peritas em mensagens comunicacionais e em ensino, segundo referencial de Fhering(15).

A segunda etapa ocorreu quarenta e cinco dias após a primeira, sendo avaliados os resultados das intervenções educativas realizadas numa visita domiciliar aos participantes. Nessa etapa, os critérios a serem julgados foram explicitados no instrumento de coleta de dados, segundo taxonomia da NOC. Cabe destacar que, também aqui, foram utilizados símbolos para favorecer a identificação visual analógica com os parâmetros disponíveis na taxonomia da NOC.

\section{RESULTADOS}

Os 25 participantes ficaram assim caracterizados: 1) 64\% eram do gênero feminino; 2) $68 \%$ casados, $20 \%$ viúvos e $12 \%$ solteiros; 3 ) a idade variou de 18 a 83 anos; 4) 88\% possuíam uma ocupação/profissão; 5) 72\% eram oriundos de cidades do interior do Estado, sendo 80\% deles naturais de cidades e municípios próximos à instituição onde os dados foram coletados; 6) Nenhum participante possuía nível superior; $48 \%$ deles não haviam completado o Ensino Fundamental, 24\% tinham o Ensino Fundamental completo, 12\%, o Ensino Médio completo e 16\% não souberam informar; 7) 96\% eram católicos e $4 \%$ afirmaram pertencer a outras denominações religiosas evangélicas.

Considerando que o(s) diagnóstico(s) emitido(s) 
pelos profissionais de saúde retrata(m) o estado de saúde de seus clientes, buscou-se caracterizar a amostra em pauta segundo os diagnósticos médicos problemas que retratam as doenças que motivaram a internação - e segundo os diagnósticos de enfermagem - problemas que retratam a reação dos participantes diante das situações de adoecimento. Foram identificados 26 diagnósticos médicos sendo os mais frequentes: doença coronariana, insuficiência renal crônica, pneumonia, hérnia de disco, nefropatia hipertensiva, epilepsia, insuficiência coronariana crônica, diabetes descompensada, infecção do trato urinário e ausência de diagnóstico definitivo durante o período de coleta de dados.

Os 84 diagnósticos de enfermagem identificados constam da tabela 1. Para evitar a inclusão de um número excessivo deles e dar visibilidade aos perfis dos problemas de enfermagem emergentes, foram priorizados os “diagnósticos nucleares" dos participantes e excluídos os "diagnósticos secundários”, os quais possuem resolução natural quando tratados os diagnósticos mais abrangentes ou nucleares a que estão vinculados. Tanto os diagnósticos médicos como os de enfermagem foram emitidos e reafirmados por profissionais peritos no uso das referidas taxonomias CID-10 ${ }^{(16)}$ e NANDA, NIC e NOC, respectivamente.

Tabela 1 - Frequência dos diagnósticos de enfermagem identificados em pacientes internados. Minas Gerais, 2008.

\begin{tabular}{lcc}
\hline Diagnóstico de Enfermagem & F & $\%$ \\
\hline Conhecimento deficiente & 21 & $25 \%$ \\
Risco para trauma vascular & 14 & $16,6 \%$ \\
Dor aguda & 7 & $8,3 \%$ \\
Déficit no autocuidado para banho/higiene & 6 & $7,14 \%$ \\
Mobilidade física prejudicada & 6 & $7,14 \%$ \\
Perfusão tissular ineficaz: cardiopulmonar & 5 & $5,95 \%$ \\
Dor crônica & 4 & $4,75 \%$ \\
Integridade da pele prejudicada & 4 & $4,75 \%$ \\
Padrão respiratório ineficaz & 4 & $4,75 \%$ \\
Risco de infecção & 4 & $4,75 \%$ \\
Risco de desequilibrio do volume de líquidos & 3 & $3,57 \%$ \\
Troca de gases prejudicada & 3 & $3,57 \%$ \\
Padrão de sono perturbado & 2 & $2,38 \%$ \\
Proteção ineficaz & 1 & $1,19 \%$ \\
\hline
\end{tabular}

A caracterização dos participantes, descrita anteriormente, reafirma a relevância da investigação do diagnóstico "conhecimento deficiente" quando se considera que os determinantes sociais refletem a posição das pessoas na hierarquia do status, do poder e no acesso aos recursos. Existem evidências que mostram que a maior parte da carga de doenças e desigualdades de saúde é influenciada por tais determinantes ${ }^{(17)}$.

Para nortear a trajetória de identificação do diagnóstico de enfermagem aplicamos a linha de raciocínio de Risner, descrita em sete fases ${ }^{(13-14)}$ e no qual são apresentados os critérios a serem utilizados para obtenção das ligações existentes entre um diagnóstico e as demais etapas do processo de enfermagem $^{(2)}$ (Quadro 1).

O diagnóstico "conhecimento deficiente”, foco desta investigação, foi identificado em $25 \%$ dos casos, sendo observados os seguintes fatores: 1) processo saúde-doença; 2) dieta prescrita; 3) procedimento/ tratamento e 4) educação para a saúde. A característica de maior incidência foi: não verbalizar adequadamente o problema ou interpretá-lo erroneamente (Quadro 2). Ao retratar situações de ausência ou deficiência de informações a respeito de uma situação de saúde, o diagnóstico "conhecimento deficiente" influencia a qualidade dos cuidados disponibilizados e os custos institucionais, uma vez que sua abordagem terapêutica é capaz de reduzir a ocorrência de comportamentos impróprios ou exagerados, favorecendo, assim, a adesão ao tratamento e melhorando o seguimento das recomendações terapêuticas dadas pela equipe multidisciplinar.

Reafirmamos a necessidade da identificação e abordagem do diagnóstico de enfermagem "conhecimento deficiente" com base em evidências identificadas em outras investigações, entre as quais destacamos: em 40\% dos participantes de uma amostra foi identificado o diagnóstico relacionado ao déficit de conhecimento sobre a doença ${ }^{(14)}$; foi unânime sua ocorrência relacionada à terapêutica e ao exame de cateterismo ${ }^{(18)}$ e foi unânime entre trabalhadores com hipertensão $\operatorname{arterial}^{(19)}$.

A seleção da conduta terapêutica torna-se mais eficiente e eficaz, do ponto de vista da solução do problema, quando é identificada e explicitada sua causa, isto é, que os fatores causadors, que podem advir não só da falta de capacidade de recordar e de interesse pelo conteúdo divulgado, como também por limitação cognitiva ou no acesso às informações, ou ainda, por interpretação errônea de informação. 
Quadro 1 - Etapas do raciocínio de Risner, 2008.

\begin{tabular}{|c|c|c|c|}
\hline \multicolumn{2}{|l|}{ Fase 1: Categorização } & \multicolumn{2}{|c|}{ Fase 2: Identificação de lacunas } \\
\hline \multicolumn{2}{|c|}{ Dados coletados segundo um referencial teórico } & \multicolumn{2}{|c|}{ Dados incompletos ou ausentes que intervêm no problema } \\
\hline Fase 3: Agrupamento & $\begin{array}{l}\text { Fase 4: Comparação entre } \\
\text { dados e referências }\end{array}$ & $\begin{array}{l}\text { Fase 5: Inferência sobre } \\
\text { problema }\end{array}$ & Fase 6: Relação causal \\
\hline $\begin{array}{l}\text { Reunião de um padrão comum, } \\
\text { ou seja, de evidências clínicas } \\
\text { ou características definidoras } \\
\text { (sinais e sintomas) a respeito } \\
\text { de uma temática. Exemplo: } \\
\text { conhecimento deficiente a } \\
\text { respeito de uma situação }\end{array}$ & $\begin{array}{l}\text { Confronto entre dados } \\
\text { identificados numa realidade } \\
\text { e as informações disponíveis } \\
\text { em suporte teóricos e } \\
\text { científicos, manuais ou } \\
\text { teorias sobre o assunto }\end{array}$ & $\begin{array}{l}\text { Inferência sobre o problema } \\
\text { de competência da } \\
\text { enfermagem, obtida do } \\
\text { confronto entre a situação } \\
\text { individual detectada e o } \\
\text { suporte teórico sobre a } \\
\text { temática }\end{array}$ & $\begin{array}{l}\text { Busca pelo fator etiológico } \\
\text { do problema ou seja, pelo } \\
\text { estabelecimento do nexo } \\
\text { causal entre problema e } \\
\text { seu(s) fator(es) } \\
\text { desencadeante(s) }\end{array}$ \\
\hline \multicolumn{4}{|c|}{ Fase 7: Impressão diagnóstica de enfermagem } \\
\hline \multicolumn{4}{|c|}{$\begin{array}{l}\text { Definição do diagnóstico de enfermagem: composição do diagnóstico de enfermagem com todos os seus elementos } \\
\text { segundo a taxonomia da NANDA }\end{array}$} \\
\hline \multicolumn{4}{|c|}{ Locus de referência para estabelecimento de ligação entre as fases do processo de enfermagem } \\
\hline \multirow[t]{2}{*}{$\begin{array}{l}\text { Planejamento da intervenção: } \\
\text { prescrição e implementação }\end{array}$} & \multicolumn{3}{|c|}{$\begin{array}{l}\text { Meta - A meta é extraída a partir do título do diagnóstico de enfermagem. No caso do } \\
\text { diagnóstico "conhecimento deficiente", será estipulado o quanto o participante alcançará o } \\
\text { antônomo do título, ou seja, um conhecimento eficiente para alcançar a saúde ou realizar } \\
\text { o tratamento com êxito }\end{array}$} \\
\hline & \multicolumn{3}{|c|}{$\begin{array}{l}\text { Definição das intervenções terapêuticas - as intervenções são extraídas a partir do } \\
\text { fator relacionado, no exemplo do diagnóstico "conhecimento deficiente". Buscam definir } \\
\text { quais as intervenções mais eficazes e eficientes para que se alcance a meta }\end{array}$} \\
\hline Resultados ou avaliação & \multicolumn{3}{|c|}{$\begin{array}{l}\text { Avaliação do êxito das intervenções implementadas - Para realizar a avaliação dos } \\
\text { resultados alcançados, é necessário realizar, subsequentemente, uma coleta de dados, } \\
\text { direcionada para as evidências que permitiram afirmar a(s) manifestação(ões)clínica(s) para } \\
\text { o diagnóstico identificado na fase de impressão diagnóstica de enfermagem e mensurar o } \\
\text { quanto ele modificou em direção à sua superação }\end{array}$} \\
\hline
\end{tabular}

A modificação da formatação do instrumento de coleta de dados para um layout predominantemente ilustrativo subsidiou as orientações a respeito das causas de "conhecimento deficiente" mostrando-se compatível com baixo nível de escolaridade dos participantes e com a busca por intervenções que melhor contemplassem a relação causal. A especificidade das intervenções foi indispensável para a abordagem das condutas terapêuticas e a superação do problema.

Para a avaliação dos resultados, foram utilizadas as escalas de medida sugeridas na NOC, ou seja, para o diagnóstico de déficit de conhecimentos observe-se o quadro 2.

Os resultados NOC, obtidos em consequência da implementação das intervenções de enfermagem
NIC, foram considerados significativos pelo teste de Wilcoxon ${ }^{(20)}$, usado para comparar os dados pareados de dois grupos: pré e pós-1 (ou seja, antes e após intervenção), permitindo identificar se houve ou não modificações significativas sobre o conhecimento dos participantes, conforme o quadro 3.

Comparando-se o conhecimento que possuíam sobre processo saúde-doença (diagnóstico 1), dieta (diagnóstico 2), procedimentos e tratamento (diagnóstico 3), educação para a saude (diagnóstico 4) com o que adquiriram após a realização da intervenção educativa e após 45 dias (Quadro 3) foi possível identificar que: houve uma melhora estatisticamente significativa em todos quando comparamos o pré-teste (antes) com o pós-teste 1(após), o que nos permite afirmar que a intervenção educativa foi relevante. 
Quadro 2 - Diagnósticos, intervenções e resultados de enfermagem segundo NANDA, NIC e NOC respectivamente relacionados ao diagnóstico de "conhecimento deficiente".

\begin{tabular}{|c|c|c|c|}
\hline \multirow[t]{2}{*}{ Diagnóstico NANDA } & \multirow[t]{2}{*}{$\begin{array}{l}\text { Intervenções } \\
\text { NIC }\end{array}$} & \multirow[t]{2}{*}{ Atividades } & $\begin{array}{l}\text { Resultados esperados } \\
\text { (NOC) }\end{array}$ \\
\hline & & & Likert** \\
\hline $\begin{array}{l}\text { Déficit de } \\
\text { conhecimento } \\
\text { relacionado ao ensino } \\
\text { do processo saúde- } \\
\text { doença, caracterizado } \\
\text { por verbalização } \\
\text { inadequada da } \\
\text { etiologia, sinais, } \\
\text { sintomas e tratamento } \\
\text { (diagnóstico 1) }\end{array}$ & $\begin{array}{l}\text { Ensino: } \\
\text { processo de } \\
\text { doença }\end{array}$ & $\begin{array}{l}\text { 1) Avaliar o atual nível de conhecimento do paciente } \\
\text { em relação ao processo específico. 2) Explicar a } \\
\text { fisiopatologia da doença e como ela se relaciona à } \\
\text { anatomia e à fisiologia. 3) Revisar o conhecimento } \\
\text { que o paciente tem sobre a sua condição. 4) } \\
\text { Descrever sinais e sintomas comuns da doença } \\
\text { quando adequado. 5) Identificar mudanças na } \\
\text { condição física do paciente. 6) Discutir mudanças no } \\
\text { estilo de vida que podem ser necessárias para } \\
\text { prevenir complicações futura e/ou controlar o } \\
\text { processo da doença. 7) Orientar pacientes sobre } \\
\text { medidas de controle/minimização dos sintomas. }\end{array}$ & $\begin{array}{l}\text { Conhecimento: } 12345 \\
\text { processo da } \\
\text { doença }\end{array}$ \\
\hline $\begin{array}{l}\text { Déficit de } \\
\text { conhecimento } \\
\text { relacionado ao ensino } \\
\text { de dieta prescrita a } \\
\text { pacientes diabéticos, } \\
\text { caracterizado por } \\
\text { verbalização } \\
\text { inadequada sobre a } \\
\text { patologia e a dieta } \\
\text { (diagnóstico 2) }\end{array}$ & $\begin{array}{l}\text { Ensino: dieta } \\
\text { prescrita }\end{array}$ & $\begin{array}{l}\text { 1) Avaliar o atual nível de conhecimento do paciente } \\
\text { sobre a dieta prescrita. 2) Explicar o propósito da } \\
\text { dieta. 3) Auxiliar o paciente a adaptar suas } \\
\text { preferências alimentares à dieta prescrita. 4) } \\
\text { Observar se a seleção alimentar do paciente está } \\
\text { adequada à dieta prescrita. 5) Reforçar informações } \\
\text { oferecidas pela equipe de saúde, quando } \\
\text { apropriado. 6) Encaminhar paciente a nutricionista } \\
\text { quando necessário. 7) Incluir família nesse processo } \\
\text { se for o caso. }\end{array}$ & $\begin{array}{l}\text { Conhecimento: } 12345 \\
\text { dieta }\end{array}$ \\
\hline $\begin{array}{l}\text { Déficit de } \\
\text { conhecimento } \\
\text { relacionado ao ensino } \\
\text { procedimento/tratame- } \\
\text { nto indicado ao } \\
\text { paciente, caracterizado } \\
\text { por verbalização } \\
\text { inadequada do que } \\
\text { consiste o } \\
\text { procedimento para } \\
\text { restabelecer a saúde } \\
\text { (diagnóstico 3) }\end{array}$ & $\begin{array}{l}\text { Ensino: } \\
\text { procedimento/ } \\
\text { tratamento }\end{array}$ & $\begin{array}{l}\text { 1) Determinar a experiência prévia e o nível de } \\
\text { conhecimento do paciente em relação ao } \\
\text { procedimento/tratamento. 2) Explicar o propósito do } \\
\text { procedimento/tratamento. 3) Explicar o } \\
\text { procedimento/tratamento. 4) Oferecer tempo ao } \\
\text { paciente para que faça perguntas e discuta suas } \\
\text { preocupações. 5) Informar o paciente sobre a data } \\
\text { e o local em que ocorrerá o procedimento }\end{array}$ & $\begin{array}{l}\text { Conhecimento: } 12345 \\
\text { procedimento } \\
\text { de tratamento }\end{array}$ \\
\hline $\begin{array}{l}\text { Déficit de } \\
\text { conhecimento } \\
\text { relacionado à } \\
\text { educação para a } \\
\text { saúde, caracterizado } \\
\text { por verbalização } \\
\text { inadequada do que } \\
\text { pode ser feito para a } \\
\text { manutenção, } \\
\text { recuperação e } \\
\text { promoção da saúde } \\
\text { (diagnóstico 4) }\end{array}$ & $\begin{array}{l}\text { Ensino: } \\
\text { indivíduo }\end{array}$ & $\begin{array}{l}\text { 1) Identificar as necessidades de aprendizagem } \\
\text { identificadas com base nas preferências. 2) Analisar } \\
\text { o acesso, a preferência do consumidor. 3) Enfatizar } \\
\text { os benefícios da saúde, imediatos ou a curto prazo a } \\
\text { serem obtidos por comportamentos de vida } \\
\text { positivos. 4) Desenvolver material educativo a um } \\
\text { nível de leitura adequado ao publico alvo. 5) } \\
\text { Envolver indivíduos da familia na implementação dos } \\
\text { planos para modificação de estilo de vida e no } \\
\text { comportamento. 6) Enfatizar a importância de } \\
\text { padrões saudáveis de alimentação, sono e } \\
\text { exercícios. }\end{array}$ & $\begin{array}{l}\text { Conhecimento: } 12345 \\
\text { promoção da } \\
\text { saúde }\end{array}$ \\
\hline
\end{tabular}

Nota: ** Indicadores Likert: 1- Nenhum; 2- Limitado; 3- Moderado; 4- Substancial; 5- Extensivo. 
Quadro 3 - Análise estatística comparativa entre pré e pós teste segundo teste de Wilcoxon.

Conhecimento após intervenções no pré-teste com o pósteste 1

Conclusão do Teste de Wilcoxon

Diagnóstico 1 Diagnóstico 2 Diagnóstico 3 Diagnóstico 4 Pré < Pós $1 *$ Pré < Pós $1 *$ Pré < Pós $1 *$ Pré < Pós $1 *$ Nota: *Significativos a $1 \%$ e $5 \%$ respectivamente.

\section{CONSIDERAÇÕES FINAIS}

A utilização das taxonomias NANDA/NIC/ NOC no processo de sistematização da assistência de enfermagem possibilitou o estabelecimento de uma ligação entre as etapas do processo; favoreceu a aplicação do método científico à práxis de enfermagem; mostrou-se factível do ponto de vista do alinhamento entre as três taxonomias; foi compatível com o referencial teórico adotado; e possibilitou a inclusão de técnicas comunicacionais e educacionais no processo de cuidar. Ao explicitar os elementos de um diagnóstico de enfermagem, o sistema NANDA norteou as etapas subsequentes para seleção da intervenção mais individualizada ao caso e avaliação dos resultados alçados dentro da abordagem terapêutica. A escolha pelo enfoque central no diagnóstico "conhecimento deficiente" mostrou-se pertinente, uma vez que esteve presente em 25\% dos diagnósticos identificados. Após a intervenção os participantes compreenderam melhor o processo saúde-doença, aumentando o conhecimento em relação ao cuidado com a doença, conhecimento da dieta, procedimentos, tratamento e conhecimento da promoção da saúde.

A aplicação de uma escala de medida contendo cinco pontos possibilitou classificar os participantes com base nos resultados obtidos e afirmar que as intervenções propostas auxiliaram o nível de conhecimento.

A ligação entre o diagnóstico e a intervenção de enfermagem favoreceu a aplicação do raciocínio diagnóstico e norteou a tomada de decisão por parte do enfermeiro, ao indicar critérios explícitos na seleção de opções de tratamento. Embora as estruturas taxonômicas NANDA, NIC e NOC tenham sido utilizadas na presente investigação, afirma-se, por experiência, que podem ser utilizadas no modelo acadêmico ou na formação em serviço para ensino da estruturação e aplicação do raciocínio clínico. A escolha pela abordagem do diagnóstico "conhecimento deficiente" mostrou-se relevante e serve de alerta para Enfermeiros com atuação clínica, uma vez que, com base nos testes estatísticos de Wilcoxon, foi possível: evidenciar o êxito de condutas terapêuticas diante de manifestações de comportamentos impróprios ou exagerados, proporcionar qualidade ao cuidado e redução dos custos institucionais, favorecer a adesão ao tratamento e melhorar o seguimento das recomendações terapêuticas da equipe multidisciplinar.

\section{REFERÊNCIAS}

1. Conselho Regional de Enfermagem do Estado de São Paulo (Coren-SP). Documentos básicos de enfermagem: principais leis e resoluções que regulamentam o exercício profissional de enfermeiros, técnicos e auxiliares de enfermagem. São Paulo:Coren;2000.

2. Arreguy-Sena C, Stuchi RAG. Boulding's theory apllied to the learing-teaching of theoretical contents and NANDA, NIC and NOC taxonomies. Conference NANDA International 2008 - [CD-ROM- article presented]. EUA: Miami 13-15 Nov 2008.

3. Garcia TR, Nóbrega MML. Processo de enfermagem: da teoria à prática assistencial e de pesquisa. Esc Anna Nery Rev Enferm. 2009 Jan/Mar;13(1):188-93.

4. Potter PA, Perry AG. Fundamentals of nursing. 7th ed. St. Louis: Mosby; 2008.

5. Arreguy-Sena, C. Processo ensino-aprendizagem das teorias de enfermagem utilizando o método comunicacional de Boulding. In: Telles-Filho, PCP, Stuchi RAG, organizadores. A enfermagem no novo milênio: uma abordagem multidisciplinar. Belo Horizonte: Difusora; 2008. p.25-38.

6. Horta WA. Processo de enfermagem. São Paulo: EPU; 2005.

7. Pérez-Rodríguez MT, Sánchez-Piña S, Franco-Orozco M, Ibarra. Aplicación del proceso de enfermería en la práctica hospitalaria y comunitaria en instituciones del Distrito Federal. Rev Enferm IMSS 2006;14(1):47-50.

8. Rodrigues MM, Souza MS, Silva JL. Sistematização da assistência de enfermagem na prevenção da lesão tecidual por pressão. Cogitare Enferm. 2008 Out/Dez;13(4):566-75.

9. NANDA - International nursing diagnoses: definitions and classification 2007-2008 Iowa (EUA): Wileyblackwell; 2008. 
10. Dochterman J, McCloskey B, Glória M. Classificação das intervenções de enfermagem (NIC). Trad. Regina Machado Garcez, $4^{\mathrm{a}}$ ed. Porto Alegre: Artmed; 2008.

11. Moorhead S, Johnson M, Maas M. Classificação dos resultados de enfermagem (NOC). Trad. Marta Avena. Porto Alegre: Artmed; 2008.

12. LoBiondo-Wod G, Haber J. Desenhos não experimentais. Rio de Janeiro: Guanabara Koogan; 2001.

13. Sena CA, Carvalho EC, Rossi LA, Rufino ME. Estratégias de implementação do processo de enfermagem para uma pessoa infectada pelo HIV. Rev Latino-Am Enfermagem. 2001 Jan;9(1):27-38.

14. Arreguy-Sena C, Gomes EAR, Cabral MIA, Centellas SV, Fonseca LN. Interrupção do uso do tabaco: consulta de enfermagem como estratégia para promoção da saúde baseada em protocolo. REME. 2006;10(3):297-305.

15. Fhering RJ. Validating diagnostic labels standardized methodology. In: Hurley ME. Classification of nursing diagnoses: proceedings of the sixth conference. St. Louis:Mosby; 1986.

16. Organização Mundial da Saúde. Classificação estatística internacional de doenças e problemas relacionados à saúde (CID-10). 7ª ed. São Paulo:Edusp; 2004.

17. Organização Mundial de Saúde. Secretaria da Comissão sobre Determinantes Sociais de Saúde. Ação sobre os determinantes sociais de saúde: aprendendo com experiências anteriores. Brasília: FIOCRUZ; 2005.

18. Pasini D, Alvim I, Kanda L, Mendes SP, Cruz D. Diagnósticos de enfermagem de pacientes internados em unidades de terapia intensiva. Rev Esc Enferm USP. 1996 Dez;30(3):501-18.

19. Pavan RMS, Siviero IMPS, Toledo VP, Duran ECM. Diagnóstico de enfermagem em trabalhadores hipertensos de uma empresa de transporte urbano coletivo. Rev Eletr Enf. [periódico na Internet]. 2005:7(2):173-8. [acesso em 2009 Mai 5] Disponível: http://www.fen.ufg.br/revista/revista7_2/pdf/original_ 04.pdf

20. Morcillo AM. Teste de Wilcoxon. Universidade de Campinas - UNICAMP, S/A. [material de apoio apostila na internet] 2006. [acesso em 2009 Mai 5]. Disponível: http://www.fcm.unicamp.br/centros/ciped/mp639/ Teste\%20de\%20Wilcoxon.pdf 